\title{
Distinguishing Hecke eigenvalues of primitive cusp forms
}

\author{
by
}

\section{J. Sengupta (Mumbai)}

Introduction. Let $f$ and $g$ be distinct primitive cusp forms of level one, i.e. normalised Hecke eigenforms on $\Gamma=\mathrm{SL}_{2}(\mathbb{Z})$ of weights $k_{1}$ and $k_{2}$ say. We write their Fourier expansions at the infinite cusp in the "standard" way as follows $(z \in \mathcal{H})$ :

$$
f(z)=\sum_{n=1}^{\infty} \lambda_{f}(n) n^{\left(k_{1}-1\right) / 2} e(n z), \quad g(z)=\sum_{n=1}^{\infty} \lambda_{g}(n) n^{\left(k_{2}-1\right) / 2} e(n z) .
$$

Here $\lambda_{f}(n)$ (resp. $\left.\lambda_{g}(n)\right)$ are the Hecke eigenvalues of $f$ (resp. $g$ ) normalised so that the standard $L$ function $L(s, f)$ (resp. $L(s, g)$ ) of $f$ (resp. $g$ ) which is given by the analytic continuation of the Dirichlet series $\sum_{n=1}^{\infty} \lambda_{f}(n) / n^{s}$ (resp. $\sum_{n=1}^{\infty} \lambda_{g}(n) / n^{s}$ ) has a functional equation under $s \mapsto 1-s$.

The strong multiplicity one theorem for GL(2) says that there are infinitely many primes $p$ such that $\lambda_{f}(p) \neq \lambda_{g}(p)$. A refinement of the strong multiplicity one theorem due to Ramakrishnan (see [15]) says that the set of primes $p$ such that $\lambda_{f}(p) \neq \lambda_{g}(p)$ has density $\geq 1 / 8$. In this article we will be interested in "finding" the smallest prime $p$ such that $\lambda_{f}(p) \neq \lambda_{g}(p)$. We will be specifically interested in the case when $k_{1} \neq k_{2}$. We will establish an upper bound $C\left(k_{1}, k_{2}\right)$ depending on $k_{1}, k_{2}$ such that for some prime $p, p \leq C\left(k_{1}, k_{2}\right)$, we must have $\lambda_{f}(p) \neq \lambda_{g}(p)$. Our motivation for this problem comes from the strong multiplicity one theorem and our result should be viewed as an effective version of the theorem in a special case. The exact statement appears below as Theorem 1.

In the third section of this paper we consider a stronger version of the problem above which is as follows. The strong multiplicity one theorem for $\mathrm{SL}(2)$ says that there are infinitely many primes $p$ such that $\left|\lambda_{f}(p)\right| \neq$ $\left|\lambda_{g}(p)\right|$. In analogy with our earlier result we will give an upper bound $\widetilde{C}\left(k_{1}, k_{2}\right)$ depending on $k_{1}, k_{2}$ such that for some prime $p, p \leq \widetilde{C}\left(k_{1}, k_{2}\right)$, we must have $\left|\lambda_{f}(p)\right| \neq\left|\lambda_{g}(p)\right|$.

2000 Mathematics Subject Classification: 11F11, 11F12. 
The plan of the article is as follows. The first section deals with notations and we also recall here some necessary background material. In the subsequent sections we state and prove our principal results, i.e. Theorems 1 and 2 .

We would like to express our gratitude to Ram Murty for discussions which led us to initiate this project. We are indebted to Jeffrey Hoffstein for his continuous encouragement and for stimulating discussions, particularly regarding Proposition 1. We thank the referee for a very thorough reading of the manuscript and for making several useful suggestions for improvement.

1. Notations. Let $k$ be a positive integer, $k$ even. $S_{k}$ will denote the space of cusp forms of weight $k$ for $\Gamma$. The subset of primitive forms in $S_{k}$, i.e. the normalised Hecke eigenforms in $S_{k}$, will be denoted by $S_{k}^{+}$. We recall that any $f \in S_{k}^{+}$has the Fourier expansion

$$
f(z)=\sum_{n=1}^{\infty} \lambda_{f}(n) n^{(k-1) / 2} e(n z), \quad z \in \mathcal{H} .
$$

Here $\lambda_{f}(n)$ is the $n$th (normalised) Hecke eigenvalue of $f, \lambda_{f}(1)=1$. The standard $L$ function of $f, L(s, f)$, is given by the Dirichlet series

$$
L(s, f)=\sum_{n=1}^{\infty} \frac{\lambda_{f}(n)}{n^{s}} .
$$

This series is absolutely convergent in $\operatorname{Re} s>1$ and has analytic continuation to the entire complex plane and after multiplication by appropriate $\Gamma$ factors satisfies a functional equation under $s \mapsto 1-s$. Let $p$ be a prime. Then we have $\lambda_{f}(p)=\alpha_{f}(p)+\beta_{f}(p)$ where $\alpha_{f}(p), \beta_{f}(p)$ are the $p$ Satake parameters of $f, \alpha_{f}(p) \beta_{f}(p)=1$. The Ramanujan-Petersson conjecture, proved by Deligne, says that

$$
\left|\alpha_{f}(p)\right|=1=\left|\beta_{f}(p)\right| .
$$

Let $f \in S_{k_{1}}^{+}$and $g \in S_{k_{2}}^{+}$be distinct and let

$$
E_{f, g}=\left\{p \text { prime } \mid \lambda_{f}(p) \neq \lambda_{g}(p)\right\}, \quad \widetilde{E}_{f, g}=\left\{p \text { prime }|| \lambda_{f}(p)|\neq| \lambda_{g}(p) \mid\right\} .
$$

The strong multiplicity one theorem for GL(2) says that the cardinality of $E_{f, g}$ is infinite. A refinement of the strong multiplicity one theorem due to Ramakrishnan ([15]) says that $E_{f, g}$ has density at least $1 / 8$. It is a consequence of the strong multiplicity one theorem for SL(2) (see [14]) that $\widetilde{E}_{f, g}$ is infinite.

We denote by $\pi_{f}$ the cuspidal automorphic representation of $\mathrm{GL}(2, \mathbb{A})$, where $\mathbb{A}$ is the adele ring of $Q$, associated to $f$. Note that $\pi_{f}$ has trivial central character. We denote by $\pi_{f}^{(2)}$ the symmetric square lift of $\pi_{f}$. This 
is an automorphic representation of $\mathrm{GL}(3, \mathbb{A})$ which is cuspidal (cf. [4]). The standard $L$ function of $\pi_{f}^{(2)}, L\left(s, \pi_{f}^{(2)}\right)$, is entire and is given in the region $\operatorname{Re} s>1$ by the absolutely convergent Dirichlet series $\sum_{n=1}^{\infty} \lambda_{f}^{(2)}(n) / n^{s}$ where

$$
\lambda_{f}^{(2)}(n)=\sum_{d^{2} \mid n} \lambda_{f}\left(\frac{n^{2}}{d^{4}}\right) .
$$

\section{Distinguishing Hecke eigenvalues of primitive forms of dis-} tinct weights. Let $f \in S_{k_{1}}^{+}$and $g \in S_{k_{2}}^{+}$with $k_{1} \neq k_{2}$. We are concerned here with the "smallest" prime $p$ such that $\lambda_{f}(p) \neq \lambda_{g}(p)$. Our result is the following. We let $k=\max \left(k_{1}, k_{2}\right)$.

TheOREM 1. Let $f$ and $g$ be as above. Then for any $\varepsilon>0$ there is a prime $p$ with $p=O_{\varepsilon}\left(k^{2+\varepsilon}\right)$ such that $\lambda_{f}(p) \neq \lambda_{g}(p)$. Here the implied constant in the $O$ symbol depends only on $\varepsilon$ and is effective.

The remainder of this section will be devoted to the proof of Theorem 1. Our basic technique for this proof and other results in this paper is the Rankin-Selberg method. The other tool is the sharp form of the PhragménLindelöf theorem due to Rademacher (see [9], [13]). We will follow here the method of Ram Murty in [11] which dealt with both forms of weight 2 and possibly different levels. In the following without loss of generality we can assume that $k_{1}>k_{2}$.

Proof of Theorem 1. We will estimate for $X>0$ the sum

$$
\sum_{n \leq X}\left(\lambda_{f}(n)-\lambda_{g}(n)\right)^{2} \log ^{2} \frac{X}{n} \text {. }
$$

This sum is equal to

$$
\sum_{n \leq X} \lambda_{f}^{2}(n) \log ^{2} \frac{X}{n}+\sum_{n \leq X} \lambda_{g}^{2}(n) \log ^{2} \frac{X}{n}-2 \sum_{n \leq X} \lambda_{f}(n) \lambda_{g}(n) \log ^{2} \frac{X}{n} .
$$

We will estimate the three summands in (2.1) individually using the Rankin-Selberg method. We have by Perron's formula, if $X$ is not an integer,

$$
\sum_{n \leq X} \lambda_{f}^{2}(n) \log ^{2} \frac{X}{n}=\frac{1}{2 \pi i} \int_{2-i \infty}^{2+i \infty}\left(\sum_{n \geq 1} \frac{\lambda_{f}^{2}(n)}{n^{s}}\right) \frac{X^{s}}{s^{3}} d s .
$$

Now the Rankin-Selberg convolution $L$ function of $\pi_{f}$ with itself is given by

$$
L(s, f \times f)=\zeta(2 s) \sum_{n=1}^{\infty} \frac{\lambda_{f}^{2}(n)}{n^{s}} \quad \text { for } \sigma=\operatorname{Re} s>1 .
$$


We can write the R.H.S. of (2.2) as

$$
\frac{1}{2 \pi i} \int_{2-i \infty}^{2+i \infty} \frac{L(s, f \times f)}{\zeta(2 s)} \frac{X^{s}}{s^{3}} d s
$$

$L(s, f \times f)$ has meromorphic continuation to the entire complex plane and its only singularity in the half-plane $\operatorname{Re} s>0$ is a simple pole at $s=1$, while $L(s, f \times g)$ is entire. We denote by $L_{\infty}(s, f \times g)$ the gamma factor that occurs in the functional equation of $L(s, f \times g)$. We have (vide [17])

$$
L_{\infty}(s, f \times g)=(2 \pi)^{-2 s} \Gamma\left(s+\frac{k_{1}-k_{2}}{2}\right) \Gamma\left(s+\frac{k_{1}+k_{2}-2}{2}\right) .
$$

This is also valid when $k_{1}=k_{2}$.

We can shift the contour of integration to the line $\operatorname{Re} s=1 / 2$. The Phragmén-Lindelöf convexity principle in conjunction with the functional equation satisfied by $L(s, f \times f), L(s, g \times g)$ and $L(s, f \times g)$ respectively allows us to estimate these functions on the line $\operatorname{Re} s=1 / 2$. This yields for any $\varepsilon>0$,

$$
\begin{gathered}
\sum_{n \leq X} \lambda_{f}^{2}(n) \log ^{2} \frac{X}{n}=\frac{L\left(\operatorname{sym}^{2} f, 1\right)}{\zeta(2)} X+O_{\varepsilon}\left(X^{1 / 2} k_{1}^{1 / 2+\varepsilon}\right), \\
\sum_{n \leq X} \lambda_{g}^{2}(n) \log ^{2} \frac{X}{n}=\frac{L\left(\operatorname{sym}^{2} g, 1\right)}{\zeta(2)} X+O_{\varepsilon}\left(X^{1 / 2} k_{2}^{1 / 2+\varepsilon}\right), \\
\sum_{n \leq X} \lambda_{f}(n) \lambda_{g}(n) \log ^{2} \frac{X}{n}=O_{\varepsilon}\left(X^{1 / 2} k^{1+\varepsilon}\right) .
\end{gathered}
$$

We will now use an important result of Goldfeld-Hoffstein-Lieman (cf. appendix of [6]) which says that there is an effective positive constant $C$ such that $L\left(\operatorname{sym}^{2} f, 1\right) \geq C / \log \left(k_{1}+1\right)\left(\operatorname{resp} . L\left(\operatorname{sym}^{2} g, 1\right) \geq C / \log \left(k_{2}+1\right)\right)$. We therefore have

$$
\sum_{n \leq X}\left(\lambda_{f}(n)-\lambda_{g}(n)\right)^{2} \log ^{2} \frac{X}{n}>0 \quad \text { for } X=O_{\varepsilon}\left(k^{2+\varepsilon}\right),
$$

i.e. $\lambda_{f}(n) \neq \lambda_{g}(n)$ for some $n, 1<n \leq X$. The Hecke eigenvalues $\lambda_{f}(n)$ are multiplicative and $\lambda_{f}\left(p^{a}\right)$ is a polynomial in $\lambda_{f}(p)$ for any positive integer $a$. We therefore have

$$
\lambda_{f}(p) \neq \lambda_{g}(p) \quad \text { for some } p=O_{\varepsilon}\left(k^{2+\varepsilon}\right) .
$$

REMARK 1. Our result remains valid when the weights of $f$ and $g$ are equal, i.e. $k_{1}=k_{2}=k$. We actually get a better result in this case, namely for any $\varepsilon>0$ there exists a prime $p, p=O_{\varepsilon}\left(k^{1+\varepsilon}\right)$, such that $\lambda_{f}(p) \neq \lambda_{g}(p)$. 
This is because we now have

$$
\sum_{n \leq X} \lambda_{f}(n) \lambda_{g}(n) \log ^{2} \frac{X}{n}=O_{\varepsilon}\left(X^{1 / 2} k^{1 / 2+\varepsilon}\right)
$$

since the convexity bound for the $L$ function $L(s, f \times g)$ on the line $\operatorname{Re} s=1 / 2$ is $O_{\varepsilon}\left((|t|+2)^{1+\varepsilon} k^{1 / 2+\varepsilon}\right)$ for all $\varepsilon>0$ in this case. However, when the weights of $f$ and $g$ are equal one knows that there is a prime $p, p=O(k)$, such that $\lambda_{f}(p) \neq \lambda_{g}(p)$.

REMARK 2. The best result one can expect here seems to be $p=O_{\varepsilon}\left(k^{\varepsilon}\right)$. This follows if we assume the Lindelöf hypothesis in the $k$ aspect for the $L$ functions $L\left(\operatorname{sym}^{2} f, s\right), L\left(\operatorname{sym}^{2} g, s\right)$ and $L(s, f \times g)$, i.e. if all these $L$ functions satisfy the estimate $O_{\varepsilon, t}\left(k^{\varepsilon}\right)$ on the line $\operatorname{Re} s=1 / 2$.

REMARK 3. In certain special cases it is possible to improve our result unconditionally, for instance if the form $g$ is kept fixed and the weight $k$ of the form $f$ tends to infinity. In this case, Sarnak ([17]) has shown that

$$
L(f \otimes g, 1 / 2+i t) \ll_{\varepsilon, t, g} k^{158 / 165} .
$$

This shows that there is a prime $p, p=O_{\varepsilon}\left(k^{316 / 165+\varepsilon}\right)$, such that $\lambda_{f}(p) \neq$ $\lambda_{g}(p)$.

REMARK 4. Moreno ([10]) has earlier proved an effective version of the strong multiplicity one theorem for cuspidal automorphic representations of $\mathrm{GL}(n)$ for any $n$. However, his result when specialised to our case yields the bound $p=O\left(k^{C(k)}\right)$ where $C(k)$ is a positive constant depending on $k$. Our result is perceptibly better.

It is appropriate to mention here that in the case of the unnormalised Hecke eigenvalues, i.e. the Fourier coefficients per se, $a(n)=\lambda_{f}(n) n^{\left(k_{1}-1\right) / 2}$ of $f$ and $b(n)=\lambda_{g}(n) n^{\left(k_{2}-1\right) / 2}$ of $g$, the problem of finding the "least" $n$ such that $a(n) \neq b(n)$ has been considered in [5], [12]. In [12] quite definitive results have been obtained which are probably best possible.

\section{Distinguishing absolute values of Hecke eigenvalues of prim-} itive forms. Let $f$ and $g$ be as in Theorem 1 . The aim of this section is to give an explicit upper bound $C(k, \varepsilon)$ say, depending on $\varepsilon$ and $k$, such that for some prime $p, p \leq C(k, \varepsilon)$, we have $\left|\lambda_{f}(p)\right| \neq\left|\lambda_{g}(p)\right|$. We denote by $F$ the symmetric square lift of $f$ and by $L(s, F \times F)$ the corresponding Rankin-Selberg $L$-function.

We will first prove a proposition which is crucial for the proof of Theorem 2. This proposition is the analogue in our context of the main result of Hoffstein and Lockhart in [6] and is interesting in its own right. We refer the reader to [6] for any hitherto unexplained notation. 
Proposition 1. Let $f \in S_{k}^{+}$and let $R_{f}$ be the residue at the pole $s=1$ of $L(s, F \times F)$. There exists a positive effective constant $\widetilde{C}$ such that

$$
R_{f} \geq \frac{\widetilde{C}}{\log (k+1)} .
$$

Proof. We will follow the method of Hoffstein-Lockhart ([6]) and also employ the very recent result of Ramakrishnan and Wang ([16]). We first observe that the Rankin-Selberg $L$ function of the symmetric square lift $F$ of $f$ with itself has a simple pole at $s=1$ as its only singularity in the half-plane $\operatorname{Re} s>0$. Hoffstein and Ramakrishnan ([7]) have shown that $L(s, F \times F)$ is given by a Dirichlet series having nonnegative coefficients. We also know that this Dirichlet series is absolutely convergent in the region $\operatorname{Re} s>1$ and is given by an Euler product there. In particular its first coefficient is 1 . We now invoke Proposition 1.1 of [6] which is tailor made for estimating the residue at $s=1$ of such Dirichlet series.

Proposition ([6]). Let $L(s)$ be an L-series of the above type, and set $R=\operatorname{Res}_{s=1} L(s)$. Let $M>1$. Suppose that $L(s)$ satisfies a growth condition on the line $\operatorname{Re} s=1 / 2$ of the form

$$
|L(1 / 2+i \gamma)| \leq M(|\gamma|+1)^{B}
$$

for some constant $B$. If $L(s)$ has no real zeros in the range

$$
1-\frac{1}{\log M}<s<1
$$

then there exists an effective constant $c=c(B)>0$ such that

$$
R^{-1} \leq c \log M
$$

In view of this proposition we need to have at our disposal two ingredients. The first is an estimate of the growth of $L(s, F \times F)$ on the line $\operatorname{Re} s=1 / 2$. This is obtained from the functional equation of $L(s, F \times F)$ via the Phragmén-Lindelöf convexity principle. The second ingredient is the absence of Landau-Siegel zeros of $L(s, F \times F)$. This is more difficult and has been very recently accomplished by Ramakrishnan and Wang. We now turn to a description of their result. We begin by observing that

$$
L(s, F \times F)=\zeta(s) L\left(\operatorname{sym}^{4} f, s\right) L\left(\operatorname{sym}^{2} f, s\right) .
$$

Kim and Shahidi ([8]) have shown that $L\left(\operatorname{sym}^{m} f, s\right)$ for $m \leq 4$ are all entire functions. Furthermore $\zeta(\sigma) \neq 0$ for $0<\sigma<1$ and Goldfeld-HoffsteinLieman (appendix of [6]) have shown that $L\left(\mathrm{sym}^{2} f, s\right)$ has no Landau-Siegel zeros. It therefore suffices to show that $L\left(\operatorname{sym}^{4} f, s\right)$ has no Landau-Siegel zeros. We now state the result of Ramakrishnan and Wang which is Theorem $\mathrm{B}^{\prime}$ of $[16]$. 
TheOREM (Ramakrishnan and Wang, [16]). Let $F$ be a number field and let $\pi$ be a cuspidal automorphic representation of $\mathrm{GL}\left(2, \mathbb{A}_{F}\right)$ with trivial central character which is not of solvable polyhedral type. Then $L\left(s, \mathrm{sym}^{4} \pi\right) a d-$ mits no Landau-Siegel zero. More explicitly, there exists a positive, effective constant $C$ such that it has no zero in the real interval $\left(1-C \mathcal{L}^{-1}, 1\right)$ where $\mathcal{L}=\log \left[N(\pi) d_{F}^{2}(2+\Lambda)^{2 N}\right]$, where $N(\pi)$ is the conductor of $\pi, N=[F: Q]$, $d_{F}$ is the discriminant of $F$ and $\Lambda$ the infinite type of $\pi$.

In our situation, $\pi=\pi_{f}$, we see that $N(\pi), d_{Q}$ and $N$ are all 1 and $\Lambda=k$. Thus $\mathcal{L}=2 \log (k+2)$. Furthermore the result of Goldfeld-Hoffstein-Lieman (see appendix of [6]) says that there exists an absolute effective constant $c$ such that $L\left(\operatorname{sym}^{2} f, s\right)$ has no zeros in the real interval $\left(1-c \mathcal{L}_{1}^{-1}, 1\right)$ where $\mathcal{L}_{1}=\log (k+1)$ in our case. Let $C_{1}=\frac{1}{2} \min (c, C / 2)$. Then $L(s, F \times F)$ has no zeros in the real interval $\left(1-C_{1} \mathcal{L}_{1}^{-1}, 1\right)$.

The sharp form of the Phragmén-Lindelöf convexity theorem ([9], [13]) yields the estimate

$$
L(1 / 2+i t, F) \ll(|t|+2)^{9 / 4+\varepsilon} k^{3 / 2+\varepsilon} \quad \forall \varepsilon>0 .
$$

This will be elaborated on later. Letting $A=\max \left(4, C_{1}^{-1}\right)$ we see that $L(s, F \times F)$ satisfies the conditions of the Proposition with $M=(k+1)^{A}$ and $B=5 / 2$ for instance. This immediately gives our assertion.

We can now state the main result of this section. We recall that $k=$ $\max \left(k_{1}, k_{2}\right)$.

Theorem 2. Let $f$ (resp. $g) \in S_{k_{1}}^{+}$(resp. $S_{k_{2}}^{+}$) where $k_{1} \neq k_{2}$. Then there is a prime $p$ such that

$$
\left|\lambda_{f}(p)\right| \neq\left|\lambda_{g}(p)\right| \quad \text { and } \quad p=O_{\varepsilon}\left(k^{4+\varepsilon}\right) \quad \text { for any } \varepsilon>0 .
$$

Proof. As in the case of Theorem 1 we can assume without loss of generality that $k_{1}>k_{2}$. We will again employ the Rankin-Selberg method, this time with the symmetric square lift of $f$ and $g$. These we denote by $F$ and $G$ respectively. Let $a(m, n)$ (resp. $b(m, n))$ denote the general Fourier coefficient of $F$ (resp. $G$ ). Then, as a Dirichlet series (cf. [6]), the Rankin-Selberg convolution of $F$ and $G$ is given by

$$
L(s, F \times G)=\zeta(3 s) \sum \frac{a(m, n) b(m, n)}{\left(m^{2} n\right)^{s}} .
$$

We will estimate

$$
\sum_{m^{2} n \leq X}(a(m, n)-b(m, n))^{2} \log ^{3} \frac{X}{m^{2} n}
$$

where $X>0$. 


$$
\begin{aligned}
\sum_{m^{2} n \leq X}^{\text {Now }}(a(m, n) & -b(m, n))^{2} \log ^{3} \frac{X}{m^{2} n} \\
= & \sum_{m^{2} n \leq X} a^{2}(m, n) \log ^{3} \frac{X}{m^{2} n}+\sum_{m^{2} n \leq X} b^{2}(m, n) \log ^{3} \frac{X}{m^{2} n} \\
& -2 \sum_{m^{2} n \leq X} a(m, n) b(m, n) \log ^{3} \frac{X}{m^{2} n} .
\end{aligned}
$$

We will estimate the three summands above individually. We have by Perron's formula, if $X \notin \mathbb{Z}$,

$$
\sum_{m^{2} n \leq X} a^{2}(m, n) \log ^{3} \frac{X}{m^{2} n}=\frac{6}{2 \pi i} \int_{2-i \infty}^{2+i \infty} \frac{L(s, F \times F)}{\zeta(3 s)} \frac{X^{s}}{s^{4}} d s .
$$

Shifting the contour of integration to the line $\operatorname{Re} s=1 / 2$, we encounter a simple pole of the integrand at $s=1$. The residue at this simple pole is $(1 / \zeta(3)) R_{f} X$. Consequently we have, for $X \notin \mathbb{Z}$,

$$
\sum_{m^{2} n \leq X} a^{2}(m, n) \log ^{3} \frac{X}{m^{2} n}=\frac{6}{\zeta(3)} R_{f} X+\frac{6}{2 \pi i} \int_{1 / 2-i \infty}^{1 / 2+i \infty} \frac{L(s, F \times F)}{\zeta(3 s)} \frac{X^{s}}{s^{4}} d s .
$$

In the following we shall need the gamma factors that enter in the functional equations of $L(s, F \times F)$ and $L(s, F \times G)$. We note here that in the case of $L(s, F \times F)$ we need only know the gamma factors that occur in the functional equations of $L\left(\operatorname{sym}^{4} f, s\right)$ and $L\left(\operatorname{sym}^{2} f, s\right)$. These are available in [3] and if we denote as before the gamma factors in $L(s, F \times F)$ by $L_{\infty}(s, F \times F)$, we have

$$
\begin{aligned}
L_{\infty}(s, F \times F)= & \pi^{-9 s / 2} \Gamma^{2}\left(\frac{s}{2}\right) \Gamma\left(\frac{s+1}{2}\right) \Gamma^{2}\left(\frac{s+k_{1}-1}{2}\right) \\
& \times \Gamma^{2}\left(\frac{s+k_{1}}{2}\right) \Gamma\left(\frac{s+2 k_{1}-2}{2}\right) \Gamma\left(\frac{s+2 k_{1}-1}{2}\right) .
\end{aligned}
$$

Using the procedure described in [3] we can also compute $L_{\infty}(s, F \times G)$. This yields

$$
\begin{aligned}
L_{\infty}(s, F \times G)= & \pi^{-9 s / 2} \Gamma\left(\frac{s}{2}\right) \Gamma\left(\frac{s+k_{1}-1}{2}\right) \Gamma\left(\frac{s+k_{1}}{2}\right) \Gamma\left(\frac{s+k_{2}-1}{2}\right) \\
& \times \Gamma\left(\frac{s+k_{2}}{2}\right) \Gamma\left(\frac{s+k_{1}+k_{2}-2}{2}\right) \Gamma\left(\frac{s+k_{1}+k_{2}-1}{2}\right) \\
& \times \Gamma\left(\frac{s+k_{1}-k_{2}}{2}\right) \Gamma\left(\frac{s+k_{1}-k_{2}+1}{2}\right) .
\end{aligned}
$$

This is also valid when $k_{1}=k_{2}$. 
The sharp form of the Phragmén-Lindelöf convexity principle (see [9], [13]) shows that for $t \in \mathbb{R}$ and any $\varepsilon>0$,

$$
\begin{aligned}
& L(1 / 2+i t, F \times F)=O_{\varepsilon}\left((|t|+2)^{9 / 4+\varepsilon} k_{1}^{3 / 2+\varepsilon}\right), \\
& L(1 / 2+i t, G \times G)=O_{\varepsilon}\left((|t|+2)^{9 / 4+\varepsilon} k_{2}^{3 / 2+\varepsilon}\right), \\
& L(1 / 2+i t, F \times G)=O_{\varepsilon}\left((|t|+2)^{9 / 4+\varepsilon} k^{2+\varepsilon}\right) .
\end{aligned}
$$

Now $1 /|\zeta(3 / 2+i t)| \leq \zeta(3 / 2)$ for all $t \in \mathbb{R}$. We therefore obtain

$$
\sum_{m^{2} n \leq X} a^{2}(m, n) \log ^{3} \frac{X}{m^{2} n}=\frac{6}{\zeta(3)} R_{f} X+O_{\varepsilon}\left(X^{1 / 2} k_{1}^{3 / 2+\varepsilon}\right) .
$$

Similarly

$$
\sum_{m^{2} n \leq X} b^{2}(m, n) \log ^{3} \frac{X}{m^{2} n}=\frac{6}{\zeta(3)} R_{g} X+O_{\varepsilon}\left(X^{1 / 2} k_{2}^{3 / 2+\varepsilon}\right)
$$

and

$$
\sum_{m^{2} n \leq X} a(m, n) b(m, n) \log ^{3} \frac{X}{m^{2} n}=O_{\varepsilon}\left(X^{1 / 2} k^{2+\varepsilon}\right) .
$$

Proposition 1 tells us that $R_{f}$ and $R_{g}$ are $\geq \widetilde{C} / \log (k+1)$ where $\widetilde{C}>0$ is an effective constant. We therefore deduce that for $X=O\left(k^{4+\varepsilon}\right)$ we must have $a(m, n) \neq b(m, n)$ for some $(m, n)$ with $m^{2} n \leq X$. The Fourier coefficients $a(m, n)$ of $F$ (resp. $b(m, n)$ of $G$ ) are multiplicative. We will show that for some $m^{\prime} \in \mathbb{N}$ with $m^{\prime} \leq X$ we must have $a\left(m^{\prime}, 1\right) \neq b\left(m^{\prime}, 1\right)$. Taking this for granted at the moment we have by multiplicativity $a\left(p^{l}, 1\right) \neq b\left(p^{l}, 1\right)$ for some prime divisor $p$ of $m^{\prime}$ and some $l \in \mathbb{N}$. If $l=1$, we are done since $a(p, 1)=a(1, p)=\lambda_{f}^{(2)}(p) \neq b(p, 1)=b(1, p)=\lambda_{g}^{(2)}(p)$ because $F$ and $G$ are self-dual cusp forms. Now if $l>1$ we deduce by self-duality of $F$ and $G$ that $a\left(p^{l}, 1\right)=a\left(1, p^{l}\right)=\lambda_{f}^{(2)}\left(p^{l}\right) \neq b\left(p^{l}, 1\right)=b\left(1, p^{l}\right)=\lambda_{g}^{(2)}\left(p^{l}\right)$. It is not difficult to see that for any $a \in \mathbb{N}, \lambda_{f}^{(2)}\left(p^{a}\right)$ is a polynomial in $\lambda_{f}^{(2)}(p)$ with integral coefficients which depend on $a$ but not on the form $f$. This can be proved by induction for instance. $\lambda_{f}^{(2)}\left(p^{l}\right) \neq \lambda_{g}^{(2)}\left(p^{l}\right)$ for some $l \in \mathbb{N}$ therefore implies that $\lambda_{f}^{(2)}(p) \neq \lambda_{g}^{(2)}(p)$. We now note that $\lambda_{f}^{(2)}(p)=\lambda_{f}\left(p^{2}\right)=\lambda_{f}^{2}(p)-1$ and similarly $\lambda_{g}^{(2)}(p)=\lambda_{g}^{2}(p)-1$. Hence we obtain $\lambda_{f}^{2}(p) \neq \lambda_{g}^{2}(p)$ and this implies that $\left|\lambda_{f}(p)\right| \neq\left|\lambda_{g}(p)\right|$ since $\lambda_{f}(p), \lambda_{g}(p)$ are real.

We will now conclude our proof by showing that $a(m, n) \neq b(m, n)$ for some $(m, n)$ with $m^{2} n \leq X$ implies that $a\left(m^{\prime}, 1\right) \neq b\left(m^{\prime}, 1\right)$ for some $m^{\prime} \in \mathbb{N}$, $m^{\prime} \leq X$. We prove this as a lemma for the convenience of the reader.

Lemma. Let $F_{1}, F_{2}$ be two self-dual cusp forms on GL(3) with Fourier coefficients $a_{1}(m, n)$ and $a_{2}(m, n)$ respectively. Suppose that for some $X>0$ 
and some pair $(m, n)$ satisfying $m^{2} n \leq X$ we have $a_{1}(m, n) \neq a_{2}(m, n)$. Then for some $m^{\prime} \in \mathbb{N}, m^{\prime} \leq X$, we have $a_{1}\left(m^{\prime}, 1\right) \neq a_{2}\left(m^{\prime}, 1\right)$.

Proof. We have (cf. [2])

$$
a_{1}(m, 1) a_{1}(1, n)=\sum_{d \mid q} a_{1}\left(\frac{m}{d}, \frac{n}{d}\right), \quad q=\operatorname{gcd}(m, n),
$$

and

$$
a_{2}(m, 1) a_{2}(1, n)=\sum_{d \mid q} a_{2}\left(\frac{m}{d}, \frac{n}{d}\right) .
$$

We are given that $a_{1}(m, n) \neq a_{2}(m, n)$. If $m$ and $n$ are coprime we have $a_{1}(m, n)=a_{1}(m, 1) a_{1}(1, n) \neq a_{2}(m, n)=a_{2}(m, 1) a_{2}(1, n)$ and hence either $a_{1}(m, 1) \neq a_{2}(m, 1)$ or $a_{1}(1, n) \neq a_{2}(1, n)$. By self-duality of $F$ and $G$, $a_{1}(1, n)=a_{1}(n, 1)$ and similarly $a_{2}(1, n)=a_{2}(n, 1)$ and since $m^{2} n \leq X$, $m^{\prime}$ will be either $m$ or $n$ and we are done. So we have to consider the case when $q>1$. This case can be subdivided into two cases.

CASE 1: For all $d \mid q, d>1$, we have $a_{1}(m / d, n / d)=a_{2}(m / d, n / d)$. In this situation we have

$$
a_{1}(m, 1) a_{1}(1, n)-a_{2}(m, 1) a_{2}(1, n)=a_{1}(m, n)-a_{2}(m, n) \neq 0
$$

and as before either $a_{1}(m, 1) \neq a_{2}(m, 1)$ or $a_{1}(n, 1) \neq a_{2}(n, 1)$.

CASE 2: For some $d \mid q, d>1$, we have $a_{1}(m / d, n / d) \neq a_{2}(m / d, n / d)$. Here if $d=q$, then $\widetilde{m}=m / q$ and $\widetilde{n}=n / q$ are coprime and as before we get either $a_{1}(\widetilde{m}, 1) \neq a_{2}(\widetilde{m}, 1)$ or $a_{1}(\widetilde{n}, 1) \neq a_{2}(\widetilde{n}, 1)$ and we are done. If now we have $1<d<q, d \mid q$, put $m_{1}=m / d, n_{1}=n / d$. Then $a_{1}\left(m_{1}, n_{1}\right) \neq$ $a_{2}\left(m_{1}, n_{1}\right)$ and $m_{1}<m, n_{1}<n$.

Iterating this procedure we arrive at a pair $\left(m_{j}, n_{j}\right), a_{1}\left(m_{j}, n_{j}\right) \neq$ $a_{2}\left(m_{j}, n_{j}\right)$ such that $m_{j}\left|m, n_{j}\right| n$ and $\operatorname{gcd}\left(m_{j}, n_{j}\right)=q_{j}$ is prime. Now,

$$
\begin{aligned}
& a_{1}\left(m_{j}, 1\right) a_{1}\left(1, n_{j}\right)=a_{1}\left(m_{j}, n_{j}\right)+a_{1}\left(m_{j} / q_{j}, n_{j} / q_{j}\right), \\
& a_{2}\left(m_{j}, 1\right) a_{2}\left(1, n_{j}\right)=a_{2}\left(m_{j}, n_{j}\right)+a_{2}\left(m_{j} / q_{j}, n_{j} / q_{j}\right) .
\end{aligned}
$$

Hence if $a_{1}\left(m_{j} / q_{j}, n_{j} / q_{j}\right)=a_{2}\left(m_{j} / q_{j}, n_{j} / q_{j}\right)$ we get

$$
a_{1}\left(m_{j}, 1\right) a_{1}\left(1, n_{j}\right)-a_{2}\left(m_{j}, 1\right) a_{2}\left(1, n_{j}\right)=a_{1}\left(m_{j}, n_{j}\right)-a_{2}\left(m_{j}, n_{j}\right) \neq 0
$$

and as before either $a_{1}\left(m_{j}, 1\right) \neq a_{2}\left(m_{j}, 1\right)$ or $a_{1}\left(n_{j}, 1\right) \neq a_{2}\left(n_{j}, 1\right)$. If $a_{1}\left(m_{j} / q_{j}, n_{j} / q_{j}\right) \neq a_{2}\left(m_{j} / q_{j}, n_{j} / q_{j}\right)$ then since $\operatorname{gcd}\left(m_{j} / q_{j}, n_{j} / q_{j}\right)=1$ we get either $a_{1}\left(m_{j} / q_{j}, 1\right) \neq a_{2}\left(m_{j} / q_{j}, 1\right)$ or $a_{1}\left(n_{j} / q_{j}, 1\right) \neq a_{2}\left(n_{j} / q_{j}, 1\right)$.

This proves the lemma and thereby concludes the proof of Theorem 2 .

REMARK 5. In the case when the weights of $f$ and $g$ are equal, i.e. $k_{1}=$ $k_{2}=k$, the situation is entirely analogous to that in Theorem 1, namely we get a better result in this case and for the same reasons. That is, given 
any $\varepsilon>0$ there is a prime $p, p=O_{\varepsilon}\left(k^{3+\varepsilon}\right)$, such that $\left|\lambda_{f}(p)\right| \neq\left|\lambda_{g}(p)\right|$ because the convexity bound for $L(s, F \times G)$ on the line $\operatorname{Re} s=1 / 2$ is $O_{\varepsilon}\left((2+|t|)^{9 / 4+\varepsilon} k^{3 / 2+\varepsilon}\right)$ in this situation. As far as the author is aware, even in the equal weight case our result is new.

REMARK 6. Moreno's result in [10] (for GL(3)) applied to our case shows that there is a prime $p$ such that $\left|\lambda_{f}(p)\right| \neq\left|\lambda_{g}(p)\right|$ and $p \leq e^{A}$ where $A$ is a positive effective constant depending on $k$. Our result is visibly better.

REMARK 7. As in the case of Theorem 1 if we assume the Lindelöf hypothesis in the $k$ aspect for $L(s, F \times F), L(s, F \times G), L(s, G \times G)$ then given any $\varepsilon>0$ there exists a prime $p, p=O_{\varepsilon}\left(k^{\varepsilon}\right)$, such that $\left|\lambda_{f}(p)\right| \neq\left|\lambda_{g}(p)\right|$.

Remark 8. Our result is true also for Maass cusp forms. The role of the weights of $f$ and $g$ are now played by the eigenvalues of $f$ (resp. $g$ ) for the hyperbolic Laplacian.

REMARK 9. The referee has kindly pointed out that F. Brumley ([1]) in a very recent preprint in the arXiv has obtained similar results for automorphic forms for $\mathrm{GL}(m)$. However, his results when specialised to the case of $\mathrm{GL}(2)$ forms are weaker than the results proved here.

\section{References}

[1] F. Brumley, Effective multiplicity one for $\mathrm{GL}_{m}$, preprint, arXiv:math.NT/0306052.

[2] D. Bump, Automorphic Forms on GL(3, $\mathbb{R})$, Lecture Notes in Math. 1083, Springer, 1984.

[3] J. Cogdell and P. Michel, On the complex moments of the symmetric power Lfunctions at $s=1$, preprint.

[4] W. Duke and E. Kowalski, A problem of Linnik for elliptic curves and mean-value estimates for automorphic representations, Invent. Math. 139 (2000), 1-39.

[5] D. Goldfeld and J. Hoffstein, On the number of Fourier coefficients that determine a modular form, in: Contemp. Math. 143, Amer. Math. Soc., 1993, 385-393.

[6] J. Hoffstein and P. Lockhart, Coefficients of Maass forms and the Siegel zero, Ann. of Math. 140 (1994), 161-181. See also the appendix of this paper by D. Goldfeld, J. Hoffstein and D. Lieman.

[7] J. Hoffstein and D. Ramakrishnan, Siegel zeros and cusp forms, Internat. Math. Res. Notices 1995, no. 6, 279-308.

[8] H. Kim and F. Shahidi, Cuspidality of symmetric powers with applications, Duke Math. J. 112 (2002), 177-197.

[9] L. Mai and M. R. Murty, The Phragmén-Lindelöf theorem and modular elliptic curves, in: Contemp. Math. 166, Amer. Math. Soc., 1994, 335-340.

[10] C. Moreno, Analytic proof of the strong multiplicity one theorem, Amer. J. Math. 107 (1985), 163-206.

[11] M. R. Murty, Bounds for congruence primes, in: Proc. Sympos. Pure Math. 66, Amer. Math. Soc., 1999, 177-192. 
[12] M. R. Murty, Congruences between modular forms, in: Analytic Number Theory, Y. Motohashi (ed.), London Math. Soc. Lecture Note Ser. 247, Cambridge Univ. Press, 1997, 309-320.

[13] H. Rademacher, On the Phragmén-Lindelöf theorem and some applications, Math. Z. 72 (1959), 192-204.

[14] D. Ramakrishnan, Modularity of the Rankin-Selberg L-series and multiplicity one for SL(2), Ann. of Math. 152 (2000), 45-111.

[15] - , A refinement of the strong multiplicity one theorem for GL(2), appendix to: l-adic representations associated to modular forms over imaginary quadratic fields, II, by R. Taylor, Invent. Math. 116 (1994), 645-649.

[16] D. Ramakrishnan and S. Wang, On the exceptional zeros of Rankin-Selberg Lfunctions, Compositio Math. 135 (2003), 211-244.

[17] P. Sarnak, Estimates for Rankin-Selberg L-functions and quantum unique ergodicity, J. Funct. Anal. 184 (2001), 419-453.

School of Mathematics

Tata Institute of Fundamental Research

Homi Bhabha Road, Colaba

Mumbai 400 005, India

E-mail: sengupta@math.tifr.res.in

Received on 17.4.2003

and in revised form on 13.11.2003 